\title{
Associations between Pneumococcal Vaccinationand Adverse Outcomes in Patients with Suspected Acute Coronary Syndrome*
}

\author{
Maliha Zahid $^{1,2}$, Ish Singla ${ }^{3,4}$, Chester B. Good ${ }^{3,5,7}$, Roslyn A. Stone ${ }^{5,6}$, Sunghee Kim ${ }^{5,6}$, \\ Michael J. Fine ${ }^{3,5,7}$, Ali F. Sonel ${ }^{3,5,7 \#}$ \\ ${ }^{1}$ Department of Developmental Biology, University of Pittsburgh, Pittsburgh, USA; ${ }^{2}$ Excelahealth Cardiology, ExcelaHealth System, \\ Greensburg, USA; ${ }^{3}$ Division of General Medicine, Department of Medicine, University of Pittsburgh, Pittsburgh, USA; ${ }^{4}$ Division of \\ Cardiology, Department of Medicine, University of Alabama, Birmingham, USA; ${ }^{5}$ Center for Healthy Equity and Research Promo- \\ tion, VA Pittsburgh Healthcare System, Pittsburgh, USA; ${ }^{6}$ Department of Biostatistics, Graduate School of Public Health, University \\ of Pittsburgh, Pittsburgh, USA; ${ }^{7}$ VA Pittsburgh Healthcare System, Pittsburgh, USA. \\ Email: \#ali.sonel@va.gov
}

Received August $2^{\text {nd }}, 2012$; revised September $3^{\text {rd }}, 2012$; accepted October $5^{\text {th }}, 2012$

\begin{abstract}
Background: Although pneumococcal vaccination prevents the most common pneumonia of bacterial etiology, its associations without comes of Acute Coronary Syndrome (ACS) are unknown. Methods: This is a prospective cohort study of 1436 patients hospitalized with suspected ACS/non-ST elevation MI that were eligible for pneumococcal vaccination. Primary outcomes were death and subsequent Myocardial Infarction (MI) within 6-months of the index hospitalization. We used Cox regression to assess associations between pneumococcal vaccination and outcomes, adjusting for influenza vaccination and relevant clinical covariates. We also utilized propensity scores to adjust for potential confounding. Results: Overall, 937 (65.3\%) patients received pneumococcal vaccination either prior to or during the index hospitalization. Unvaccinated patients had higher mortality $(26.9 \%$ vs $7.9 \% ; p<0.001)$ and non-significantly higher frequency of subsequent MI (7.4\% vs $3.5 \% ; \mathrm{p}=0.06)$.Compared to patients who did not receive either pneumococcal or influenza vaccination, the unadjusted Hazard Ratio (HR) of death was significantly lower for those who received only pneumococcal vaccination $(\mathrm{HR}=0.13 ; 95 \% \mathrm{CI} 0.07-0.23)$ or both vaccinations $(\mathrm{HR}=0.66,95 \% \mathrm{CI} 0.47-0.92)$, and significantly higher for patients who received only influenza vaccination $(\mathrm{HR}=1.88,95 \%$ CI $1.33-2.64)$. The corresponding HRs and 95\% CIs for subsequent MI were 0.58 (95\% CI $0.32-1.03)$ for pneumococcal vaccination only, $0.41(95 \%$ CI $0.21-0.80)$ for both vaccinations and 0.97 (95\% CI $0.48-1.95)$ for influenza vaccination alone. These remained unchanged after covariate or propensity score adjustment. Conclusions: Among patients hospitalized with suspected ACS, pneumococcal vaccination, with or without influenza vaccination, was associated with significantly lower risk of mortality within 6 months.
\end{abstract}

Keywords: Pneumococcal Vaccination; Acute Coronary Syndromes; Outcomes

\section{Introduction}

Cardiovascular disease is the leading cause of mortality in United States. Current guidelines emphasize primary and secondary prevention of morbidity and mortality of cardiovascular disease through treatment of modifiable risk factors, such as tobacco use, diabetes, hypertension, and hypercholesterolemia. One major morbid complication of atherosclerosis is plaque rupture or instability leading to an Acute Coronary Syndrome (ACS) or Myocar-

\footnotetext{
*Funding: This study was funded by a Competitive Pilot Project Fund award from the Department of Veterans Affairs, Veterans Integrated Service Network (VISN) 4

${ }^{\#}$ Corresponding author.
}

dial Infarction (MI). Observational studies have shown an increase in the incidence and mortality of acute MI in winter months consistent with a potential role of microbial agents acting as inflammatory stimuli [1,2]. In addition, one large study demonstrated an increased short term risk of MI or stroke following respiratory infections [3]. Although the role of inflammation in the pathophysiology of atherosclerotic disease is becoming better recognized [4,5], the mechanisms by which infections potentially precipitate acute coronary events are not well-understood.

The role of the immune system in the pathogenesis of atherosclerosis is relatively complex, potentially induc- 
ing both atherogenic and atheroprotective mechanisms [6-8]. Novel approaches to prevent cardiovascular diseases include antibiotic therapy and vaccination for potential pathogens [9]. Although antibiotic use has not been shown to be effective $[9,10]$, recent studies have demonstrated that influenza vaccination is protective for MI and cardiac arrest in patients with documented coronary heart disease and in patients presenting with an ACS [11,12]. Pneumococcal vaccination has been shown to have an anti-atherogenic effect in animal studies [13], which may be mediated by decreasing Low Density Lipoprotein (LDL) accumulation in arteries. However, a reduction in LDL accumulation in arteries associated with pneumococcal vaccination has not been demonstrated in humans.

Although pneumococcal vaccination effectively reduces the most common cause of bacterial pneumonia due to streptococcus pneumonia [14-16] and cardiovascular complications occur more commonly following pneumonia $[17,18]$, the association between pneumococcal vaccination and adverse outcomes in patients with ACS remains largely unexplored. One case-control study showed that patients presenting with a MI were $50 \%$ less likely to have received pneumococcal vaccination compared to patients admitted with non-cardiac diagnoses [19]. However, the case-control design did not allow assessment of the association of pneumococcal vaccination and subsequent mortality. In contrast, Tseng et al. failed to find an association between receipt of pneumococcal vaccination and risk of MI or stroke in men [20]. Because the few published studies of a possible role of pneumococcal vaccination in cardiovascular events have reported conflicting results, we sought to examine the associations between pneumococcal vaccination and subsequent adverse outcomes in patients hospitalized with suspected ACS/ non-ST elevation myocardial infarction (ACS/NSTEMI) at a tertiary care VA Medical Center.

\section{Methods}

We conducted a prospective cohort study of patients admitted to the VA Pittsburgh Healthcare System with suspected ACS/NSTEMI on the basis of chest pain, suspected unstable angina or anginal-equivalent between January 2001 and April 2006. Patients were followed for 6 months from the index admission date to assess death and subsequent MI. The independent variable of interest was pneumococcal vaccination, with influenza vaccine status and other baseline patient characteristics considered as potential confounders. The study was approved by the VA Pittsburgh Institutional Review Board.

Of 1531 patients presenting with a suspected or definitive diagnosis of ACS/NSTEMI, the 1436 with an indication for pneumococcal vaccination based on CDC guidelines were included in the analysis [21]. The primary indication for pneumococcal vaccination utilized in this study was age $\geq 65$ years. Among adults younger than 65 years, presence of chronic pulmonary disease (i.e., chronic bronchitis, emphysema, asthma or history of smoking) or risk factors for invasive pneumococcal disease (i.e., splenectomy or sickle cell disease) were considered indications for pneumococcal vaccination. ACS/NSTEMI was ascertained by measurement of troponin-I levels obtained in the emergency department or within 6 hours of admission. We excluded patients who had ST elevations persisting $>10$ minutes or who were transferred from another medical facility. For patients who met study inclusion criteria on more than one occasion, we included only their first ("index") admission.

\subsection{Baseline Data Collection}

We reviewedpatient electronic medical records from the study hospital andall 9 affiliated VA Hospitals in its referral network. Baseline data consisted of demographic information, history and physical examination findings, laboratory results (including cardiac biomarkers), medications and non-pharmacologic therapies (on arrival, within 48 hours of admission and/or at discharge), and echocardiographic findings. The presence or absence of dynamic ST depression was ascertained by a boardcertified cardiologist (AFS) without knowledge of patient baseline clinical information or medical outcomes. Pulmonary edema was considered to be present if documented in the staff radiologist's reading of the admission chest x-ray. Elevated troponin-I was defined according to the manufacturer's recommended cutoff values for myocardial injury. All laboratory analyses were performed using standard, automated procedures with commercial kits in a single laboratory. All treatment decisions were made at the discretion of the responsible VA medical providers.

We extracted data from VA electronic medical records on prior pneumococcal vaccination at any time, influenza vaccination within 12 months prior to admission, and both vaccinations during the index hospitalization and during the 6 month follow-up period. Patients with no documented record of vaccination were considered to be unvaccinated.

\subsection{Assessment of Study Outcomes}

We ascertained study outcomes by reviewing the electronic medical records from the study hospital and the 9 affiliated VA hospitals. We also reviewed VA national databases that include records of all hospital admissions to VA facilities and applications for Veteran death benefits.

The primary outcomes were death from any cause and 
MI within 6 months of the index admission date. Subsequent MI was defined as a typical rise and fall of biochemical markers of myocardial necrosis along with at least one of the following: ischemic cardiac symptoms, development of pathological Q waves on the ECG, ECG changes indicative of cardiac ischemia and/or an acute coronary artery intervention remote from the initial presenting event, as defined by the American College of Cardiology [22].

\subsection{Statistical Analysis}

Because of potential confounding by influenza vaccination status, we compared baseline clinical, laboratory, and treatment characteristics by pneumococcal and influenza vaccination status at admission (considered jointly), using chi-square tests for discrete variables and analysis of variance for continuous variables. In addition, we compared these baseline characteristics for three patient subgroups defined by time-dependent pneumococcal vaccination status (i.e. vaccinated prior to hospitalization, vaccinated during or after hospitalization, and vaccination not documented). We also compared the characteristics of the two combined pneumococcal vaccination subgroups to the presumed unvaccinated group, using logistic regression for binary characteristics and linear regression for continuous variables.

We summarized death and subsequent MI by timedependent pneumococcal and influenza vaccination status (considered jointly). Because troponin values are established predictors of subsequent adverse cardiovascular events, we also summarized these data separately for patients with and without troponin elevation. We used Fisher exact tests to compare outcomes by pneumococcal and influenza vaccination status (considered jointly) and by each type of vaccination separately.

In time to event analyses, we considered pneumococcal and influenza vaccination status as time-dependent predictors. Kaplan-Meier curves summarized time to death and subsequent MI by pneumococcal and influenza vaccination status (considered jointly). We used Cox regression to model the associations between pneumococcal and influenza vaccination and patient outcomes, considering univariate and main effect models. Preliminary analyses indicated a significant interaction between pneumococcal and influenza vaccination in the mortality analysis $(\mathrm{p}=0.005)$ but not in the MI analysis $(\mathrm{p}=0.53)$. We report interaction model sum adjusted and adjusted for the clinical covariates shown in Table 1, using a forward selection approach with a p-value $<0.10$ as the entry criterion. In each model, the corresponding reference group is designated with a HR of 1.0. Two-tailed p-values
$<0.05$ were considered statistically significant.

We used logistic regression to develop two separate propensity scores to model the predicted probabilities of receiving 1) pneumococcal vaccination and 2) influenza vaccination as functions of the covariates shown in Table 1. The propensity score models for pneumococcal vaccination and influenza vaccination demonstrated modest discrimination (c statistics of 0.66 and 0.60 , respectively) and no evidence of lack of fit ( $p=0.90$ and $p=$ 0.60 , respectively). To adjust for each type of selection bias using propensity scores, we also stratified analyses of patient outcomes by quintile of each propensity score separately. To assess the extent to which the propensity score reduced observed covariate imbalances, we also compared patient clinical, laboratory, and treatment characteristics for patients who did and did not receive the pneumococcal vaccination, stratified on quintiles of the propensity score for pneumococcal vaccination.

\section{Results}

The study population was predominantly white $(78.2 \%)$ and male $(97.7 \%)$, with a mean age of 67.3 years (s.d. 11.8 years) and corresponding median age of 69 years (inter-quartile range 58 - 77 years). At admission 59.7\% of the 1436 eligible patients had received pneumococcal vaccination and $35.0 \%$ had received influenza vaccination in the past 12 months; 350 (24.4\%) received both vaccinations, $507(35.3 \%)$ received pneumococcal vaccination only, $153(10.7 \%)$ received influenza vaccination only, and 426 (29.7\%) had received neither vaccination (Table 1). Patients who had received neither vaccination prior to admission tended to be younger, less likely to have coronary artery disease or congestive heart failure, and less likely to use statins even though their mean total cholesterol, LDL, and triglycerides were significantly higher than those of the other three vaccination subgroups.

An additional 80 patients (5.6\%) received pneumococcal vaccination during or after admission (Table 2). Among those with documented pneumococcal vaccination, the median duration between vaccination and the index admission was 2.4 years (inter-quartile range 9 months to 4.2 years).Patients with a pneumococcal vaccination at any time were more likely to have diabetes mellitus and/ or hypertension than were patients who had not received pneumococcal vaccination.A total of 347 patients had a CABG and 60 had a PTCA, with no significant differences by pneumococcal vaccination status. Except for influenza vaccination, which was not included in the propensity score for pneumococcal vaccination, propensity score adjustment eliminated previously significant 
Table 1. Patient clinical, laboratory, and treatment characteristics by pneumococcal and influenza vaccination status at admission ( $N$ 1436).

\begin{tabular}{|c|c|c|c|c|c|}
\hline \multicolumn{6}{|c|}{ Pneumococcal and influenza vaccination status at admission } \\
\hline Characteristic & $\begin{array}{c}\text { Pneumococcal and } \\
\text { influenza } \\
(\mathrm{N}=350) \mathbf{n} / \mathbf{N}(\%)^{*}\end{array}$ & $\begin{array}{c}\text { Pneumococcal } \\
\text { no influenza } \\
(\mathrm{N}=507) \mathbf{n} / \mathbf{N}(\%)^{*}\end{array}$ & $\begin{array}{c}\text { No } \\
\text { pneumococcal } \\
\text { influenza } \\
(\mathbf{N}=153) \\
\mathbf{n} / \mathbf{N}(\%)^{*}\end{array}$ & $\begin{array}{c}\text { No } \\
\text { pneumococcal } \\
\text { no influenza } \\
(\mathrm{N}=426) \\
\mathrm{n} / \mathrm{N}(\%)^{*}\end{array}$ & $\begin{array}{c}\text { Unadjusted } \\
\text { p-value }^{\dagger}\end{array}$ \\
\hline \multicolumn{6}{|l|}{ Demographics } \\
\hline Age (Mean \pm S.D.) & $69.8 \pm 10.7$ & $68.9 \pm 11.1$ & $68.5 \pm 12.2$ & $62.9 \pm 12.1$ & $<0.001$ \\
\hline Race (white) & $281 / 349(80.5)$ & $402 / 506(79.5)$ & $116 / 153(75.8)$ & $320 / 423(75.7)$ & 0.30 \\
\hline Sex (male) & $343(98.0)$ & $496(97.8)$ & $146(95.4)$ & $418(98.1)$ & 0.26 \\
\hline \multicolumn{6}{|l|}{ Comorbidity } \\
\hline Diabetes mellitus & $174(49.7)$ & $212(41.8)$ & $57(37.3)$ & $157(36.9)$ & $<0.01$ \\
\hline Hypertension & $270(77.1)$ & $372(73.4)$ & $111(72.6)$ & $268(62.9)$ & $<0.001$ \\
\hline Coronary artery disease & $212(60.6)$ & $275(54.2)$ & $80(52.3)$ & $197(46.2)$ & $<0.001$ \\
\hline History of myocardial infarction & $77(22.0)$ & $126(24.9)$ & $38(24.8)$ & 88 (20.7) & 0.43 \\
\hline Congestive heart failure & $82(23.4)$ & $91(18.0)$ & $34(22.2)$ & $58(13.6)$ & $<0.01$ \\
\hline Cerebrovascular accident & $40(11.4)$ & $75(14.8)$ & $14(9.2)$ & $42(9.9)$ & 0.08 \\
\hline Chronic renal insufficiency & $63(18.0)$ & $85(16.8)$ & $36(23.5)$ & $61(14.3)$ & 0.07 \\
\hline Current or past smoker & $223(63.7)$ & $343(67.7)$ & $117(76.5)$ & $305(71.6)$ & 0.02 \\
\hline \multicolumn{6}{|l|}{ Medication use } \\
\hline Statin use ${ }^{\S}$ yes & $187(53.4)$ & $268(52.9)$ & $73(47.7)$ & $186(43.7)$ & 0.01 \\
\hline Missing & $47(13.4)$ & $56(11.0)$ & $26(17.0)$ & $80(18.8)$ & \\
\hline Aspirin & $289 / 350(82.6)$ & $415 / 506(82.0)$ & $132 / 153(86.3)$ & $349 / 426(81.9)$ & 0.64 \\
\hline Clopidogrel/Ticlodipine & $50(14.3)$ & $104(20.5)$ & $25(16.3)$ & $80(18.8)$ & 0.12 \\
\hline Heparin & $4(1.1)$ & $28(5.5)$ & $9(5.9)$ & $14(3.3)$ & $<0.01$ \\
\hline Glycoprotein $2 \mathrm{~b}-3 \mathrm{a}$ inhibitor & $18(5.1)$ & $46(9.1)$ & $13(8.5)$ & $44(10.3)$ & 0.07 \\
\hline Beta blocker & $225 / 303(74.3)$ & $323 / 451(71.6)$ & $89 / 127(70.1)$ & $241 / 346(69.7)$ & 0.61 \\
\hline Ace inhibitor & $191 / 303(63.0)$ & $268 / 450(59.6)$ & $70 / 127(55.1)$ & $185 / 346(53.5)$ & 0.07 \\
\hline \multicolumn{6}{|l|}{ Clinical and laboratory findings } \\
\hline Systolic blood pressure $<90 \mathrm{~mm} \mathrm{Hg}$ & $60(17.1)$ & $75(14.8)$ & $27(17.7)$ & $71(16.7)$ & 0.74 \\
\hline Pulmonary edema on admission & $41(11.7)$ & $37(7.3)$ & $21(13.7)$ & $42(9.9)$ & 0.05 \\
\hline Hemoglobin $<11.5 \mathrm{gm} / \mathrm{dl}$ & $93 / 350(26.6)$ & $116 / 506(22.9)$ & $47 / 153(30.7)$ & $94 / 425(22.1)$ & 0.11 \\
\hline $\begin{array}{c}\text { Left ventricular } \\
\text { ejection fraction }<35 \%\end{array}$ & $47(13.4)$ & $60(11.8)$ & $20(13.1)$ & $72(16.9)$ & 0.18 \\
\hline Missing & $124(35.4)$ & $196(38.7)$ & $46(30.1)$ & $145(34.0)$ & \\
\hline Troponin elevated & $83(23.7)$ & $96(18.9)$ & $39(25.5)$ & $110(25.8)$ & 0.06 \\
\hline
\end{tabular}




\begin{tabular}{|c|c|c|c|c|c|}
\hline Cholesterol profile & Mean \pm S.D. & Mean \pm S.D. & Mean \pm S.D. & Mean \pm S.D. & \\
\hline Total cholesterol (mg/dl) & $157.9 \pm 43.0$ & $162.3 \pm 47.4$ & $154.9 \pm 52.4$ & $170.7 \pm 53.7$ & $<0.01$ \\
\hline $\operatorname{LDL}(\mathrm{mg} / \mathrm{dl})$ & $95.5 \pm 38.7$ & $98.0 \pm 39.5$ & $93.6 \pm 41.5$ & $105.6 \pm 45.2$ & $<0.01$ \\
\hline $\operatorname{HDL}(\mathrm{mg} / \mathrm{dl})$ & $37.7 \pm 14.7$ & $36.9 \pm 13.6$ & $37.1 \pm 14.7$ & $37.8 \pm 35.6$ & 0.95 \\
\hline Triglycerides (mg/dl) & $132.0 \pm 80.6$ & $147.5 \pm 160.1$ & $147.8 \pm 138.8$ & $163.6 \pm 151.8$ & 0.05 \\
\hline \multicolumn{6}{|l|}{ Invasive medical procedures } \\
\hline Coronary artery bypass graft (CABG) & $95(27.1)$ & $126(24.9)$ & $41(26.8)$ & $85(20.0)$ & 0.09 \\
\hline $\begin{array}{l}\text { Percutaneous transluminal } \\
\text { coronary angioplasty (PTCA) }\end{array}$ & $10(2.9)$ & $25(4.9)$ & $2(1.3)$ & $23(5.4)$ & 0.07 \\
\hline
\end{tabular}

S.D. denotes standard deviation; $\mathrm{N}$ denotes the denominator or the total number of patients in a sub-group or with a given characteristic assessed; $\mathrm{n}$ denotes the numerator, or the number of patients with a given characteristic present; ${ }^{*}$ Percent (\%) was calculated by dividing $\mathrm{n}$ by $\mathrm{N}$ for each characteristic. Denominators (N) are recorded for all characteristics with more than 1 missing observation for the 4 pneumococcal/influenza vaccination status subgroups. The overall sample sizes for cholesterol profile values are: total cholesterol $(\mathrm{N}=1150)$, LDL $(\mathrm{N}=1155)$, HDL $(\mathrm{N}=1157)$ and triglycerides $(\mathrm{N}=1132)$; ${ }^{\dagger}$ Based on 3 degree of freedom chi-square tests for categorical variables and analysis of variance for continuous variables (to compare the 4 pneumococcal/influenza vaccination subgroups); ${ }^{\S}$ Based on a 6 degree of freedom chi-square test to compare the 4 pneumococcal/influenza vaccination subgroups with respect to categories of statin use (yes, no, missing).

differences by pneumococcal vaccination status (Table 2). About one-quarter $(25.2 \%)$ of patients received influenza vaccination during or after admission (Table 2). Among the 937 patients who received pneumococcal vaccination, 667 (71.2\%) also received influenza vaccination; $301(60.3 \%)$ of the 499 patients without documented pneumococcal vaccination also had no documented influenza vaccination (Table 2). Given that a patient received an influenza vaccination, the estimated odds ratio of receiving pneumococcal vaccination was $3.8(95 \% \mathrm{CI}$; $3.0-4.7)$.

\subsection{Unadjusted Associations between Vaccination and Patient Outcomes}

Overall, 208 patients (14.5\%) died within 6 months (Table 3$)$. Mortality was significantly lower in patients receiving pneumococcal vaccination prior to, or during or after, admissioncompared to unvaccinated patients $(8.3 \%$, $3.8 \%$ and $26.9 \%$, respectively; $\mathrm{p}<0.001$ ).

Mortality was higher among patients who received influenza vaccination prior to admission compared to those who received it during or after admission or were unvaccinated $(20.9 \%, 4.4 \%$ and $15.2 \%$, respectively; $\mathrm{p}<0.001)$. The highest mortality rates were observed among patients with no pneumococcal vaccination who either had influenza vaccination prior to admission $(37.7 \%)$ or did not receive influenza vaccination $(24.9 \%)$. Similar patterns of mortality by pneumococcal and influenza vaccination status were observed across subgroups of patients with and without elevated troponin levels, although the corresponding mortality rates were higher among patients with laboratory evidence of MI at admission (data not shown).

A total of 70 patients (4.9\%) developed subsequent MIs within 6 months (Table 3), including 19 of the 208 (9.1\%) who died within 6 months. The frequency of subsequent MI was significantly lower in patients who received pneumococcal vaccination prior to, or during or after, admission compared to unvaccinated patients $(3.5 \%$, $3.8 \%$, and $7.4 \%$, respectively; $\mathrm{p}<0.01$ ). The rate of subsequent MI did not differ significantly by influenza vaccination status $(\mathrm{p}=0.32)$.

Univariate Cox model analyses of mortality with timedependent vaccination status indicated a significantly reduced Hazard Ratio (HR) of 0.31 associated with pneumococcal vaccination (relative to no documented pneumococcal vaccination) and a significantly increased HR $=1.90$ associated with influenza vaccination (relative to no documented influenza vaccination; $p<0.001$ for each; Table 4). In univariate Cox models for subsequent MI, pneumococcal vaccination was associated with a significantly reduced HR of $0.50(\mathrm{p}<0.01)$ and influenza vaccination was associated with a non-significantly reduced HR of $0.73(p=0.22)$. For both outcomes, similar HRs were estimated when each vaccination was adjusted for the other in the corresponding main effects model (e.g., patients with pneumococcal vaccination were compared to patients of the same influenza vaccination status who did not have documented pneumococcal vaccination).

As shown in the Kaplan-Meier curves (Figure 1(a)) and summarized in Table 4, patients with pneumococcal vaccination only or both vaccinations had significantly lower mortality compared to patients with neither vaccination, with an unadjusted HR of 0.13 (95\% CI $0.07-0.23)$ for 

Patients with Suspected Acute Coronary Syndrome

Table 2. Patient clinical, laboratory, and treatment characteristics by pneumococcal vaccination status $(\mathrm{N}=1436)$.

\begin{tabular}{|c|c|c|c|c|c|}
\hline \multirow[b]{2}{*}{ Characteristic } & \multicolumn{3}{|c|}{ Pneumococcal vaccination status } & \multirow[b]{2}{*}{$\begin{array}{c}\text { Unadjusted } \\
\text { p-value }^{\dagger}\end{array}$} & \multirow[b]{2}{*}{$\begin{array}{c}\text { Pneumococcal } \\
\text { vaccine propensity } \\
\text { score adjusted } \\
\text { p-value }\end{array}$} \\
\hline & $\begin{array}{c}\text { Prior to } \\
\text { admission } \\
(\mathbf{N}=857) \\
\mathbf{n} / \mathbf{N}(\%)^{*}\end{array}$ & $\begin{array}{c}\text { During or after } \\
\text { admission } \\
(\mathbf{N}=\mathbf{8 0}) \\
\mathbf{n} / \mathbf{N}(\%)^{*}\end{array}$ & $\begin{array}{l}\text { Vaccinationnot } \\
\text { documented } \\
(\mathrm{N}=499) \\
\mathrm{n} / \mathrm{N}(\%)^{*}\end{array}$ & & \\
\hline \multicolumn{6}{|l|}{ Demographics } \\
\hline Age (Mean \pm S.D. $)$ & $69.2 \pm 10.9$ & $64.6 \pm 11.1$ & $64.3 \pm 12.6$ & $<0.001$ & 0.09 \\
\hline Race (white) & $683 / 855(79.9)$ & $60 / 80(75.0)$ & $376 / 496(75.8)$ & 0.17 & 0.76 \\
\hline Sex (male) & $839(97.9)$ & $78(97.5)$ & $486(97.4)$ & 0.83 & 0.71 \\
\hline \multicolumn{6}{|l|}{ Comorbidity } \\
\hline Diabetes mellitus & $386(45.0)$ & $35(43.8)$ & $179(35.9)$ & $<0.01$ & 0.70 \\
\hline Hypertension & $642(74.9)$ & $57(71.3)$ & $322(64.5)$ & $<0.001$ & 0.90 \\
\hline Coronary artery disease & $487(56.8)$ & $37(46.3)$ & $240(48.1)$ & $<0.01$ & 0.84 \\
\hline History of myocardial infarction & $203(23.7)$ & $14(17.5)$ & $112(22.4)$ & 0.43 & 0.27 \\
\hline Congestive heart failure & $173(20.2)$ & $5(6.3)$ & $87(17.4)$ & $<0.01$ & 0.99 \\
\hline Cerebrovascular accident & $115(13.4)$ & $9(11.8)$ & $47(9.4)$ & 0.09 & 0.92 \\
\hline Chronic renal insufficiency & $148(17.3)$ & $11(13.8)$ & $86(17.2)$ & 0.72 & 0.93 \\
\hline Current or past smoker & $566(66.0)$ & $60(75.0)$ & $362(72.6)$ & 0.02 & 0.97 \\
\hline \multicolumn{6}{|l|}{ Medication Use } \\
\hline $\begin{array}{l}\text { Statin use } \\
\text { yes }\end{array}$ & $455(53.1)$ & $33(41.3)$ & $226(45.3)$ & $<0.1$ & 0.96 \\
\hline Missing & $103(12.0)$ & $18(22.5)$ & $88(17.6)$ & & \\
\hline Aspirin & $704 / 856(82.2)$ & $69 / 80(86.3)$ & $412 / 499(82.6)$ & 0.67 & 0.31 \\
\hline Clopidogrel/Ticlodipine & $154(18.0)$ & $16(20.0)$ & $89(17.8)$ & 0.89 & 0.81 \\
\hline Heparin & $32(3.7)$ & $2(2.5)$ & $21(4.2)$ & 0.74 & 0.38 \\
\hline Glycoprotein $2 b-3 a$ inhibitor & $64(7.5)$ & $9(11.3)$ & $48(9.6)$ & 0.25 & 0.44 \\
\hline Beta blocker & $548 / 754(72.7)$ & $43 / 62(69.4)$ & $287 / 411(69.8)$ & 0.54 & 0.76 \\
\hline Ace inhibitor & $459 / 753(61.0)$ & $33 / 62(53.2)$ & $222 / 411(54.0)$ & 0.05 & 0.41 \\
\hline \multicolumn{6}{|l|}{ Clinical and laboratory findings } \\
\hline Systolic blood pressure $<90 \mathrm{~mm} \mathrm{Hg}$ & $135(15.8)$ & $10(12.5)$ & $88(17.6)$ & 0.43 & 0.75 \\
\hline Pulmonary edema on admission & $78(9.1)$ & $6(7.5)$ & $57(11.4)$ & 0.30 & 0.80 \\
\hline Hemoglobin $<11.5 \mathrm{gm} / \mathrm{dl}$ & $209 / 856(24.4)$ & $17 / 80(21.3)$ & $124 / 498(24.9)$ & 0.78 & 0.88 \\
\hline Left ventricular ejection fraction $<35 \%$ & $107(12.5)$ & $8(10.0)$ & $84(16.8)$ & 0.10 & 0.97 \\
\hline Missing & $320(37.3)$ & $26(32.5)$ & $165(33.1)$ & & \\
\hline Troponin elevated & 179 (20.9) & $19(23.8)$ & $130(26.1)$ & 0.09 & 0.73 \\
\hline Cholesterol profile & Mean \pm S.D. & Mean \pm S.D. & Mean \pm S.D. & & \\
\hline Total cholesterol (mg/dl) & $160.5 \pm 45.7$ & $165.1 \pm 46.7$ & $166.8 \pm 54.9$ & 0.12 & 0.29 \\
\hline $\mathrm{LDL}(\mathrm{mg} / \mathrm{dl})$ & $96.9 \pm 39.2$ & $97.8 \pm 36.8$ & $103.2 \pm 45.7$ & 0.05 & 0.14 \\
\hline
\end{tabular}




\section{Continued}

$\begin{array}{cccccr}\text { HDL }(\mathrm{mg} / \mathrm{dl}) & 37.2 \pm 14.1 & 36.5 \pm 13.4 & 37.8 \pm 33.4 & 0.88 & 0.85 \\ \text { Triglycerides (mg/dl) } & 141.1 \pm 133.5 & 152.3 \pm 103.9 & 160.6 \pm 155.0 & 0.09 & 0.11\end{array}$

Invasive Medical Procedures

Coronary Artery Bypass Graft (CABG)

$221(25.8)$

$15(18.8)$

$5(6.3)$

$42(52.5)$

$260(30.3)$

$23(28.8)$

Percutaneous Transluminal

Coronary Angioplasty (PTCA)

$247(28.8)$
$111(22.2)$

$20(4.0)$

0.17

0.97

0.65

$138(27.7)$

$<0.001^{\S}$

$<0.001$

$60(12.0)$

$301(60.3)$

S.D. denotes standard deviation; $\mathrm{N}$ denotes the denominator or the total number of patients in a sub-group or with a given characteristic assessed; $\mathrm{n}$ denotes the numerator, or the number of patients with a given characteristic present; ${ }^{*}$ Percent (\%) was calculated by dividing $\mathrm{n}$ by Nfor each characteristic. Denominators (N) are recorded for all characteristics with more than 1 missing observation for the 3 pneumococcal vaccination status subgroups. The overall denominators for cholesterol profile values are: total cholesterol $(\mathrm{N}=1150)$, LDL $(\mathrm{N}=1155)$, HDL $(\mathrm{N}=1157)$ and triglycerides $(\mathrm{N}=1132)$; ${ }^{\dagger}$ Based on 2 degree of freedom chi-square tests for categorical variables and analysis of variance for continuous variables(to compare the 3 pneumococcal vaccination subgroups); ${ }^{\dagger}$ Based on 1 degree of freedom chi-square tests, using logistic regression or linear regression (as appropriate) to compare characteristics for patients receiving any pneumococcal vaccination vs none; ${ }^{\S}$ Based on a 4 degree of freedom chi-square test to compare the 3 pneumococcal vaccination subgroups with respect to categories of statin use (yes, no, missing) or influenza vaccination status.

Table 3. Outcomes at 6 months by pneumococcal and influenza vaccination status considered jointly $(\mathrm{N}=1436)$.

\begin{tabular}{|c|c|c|c|c|c|}
\hline \multicolumn{6}{|c|}{ Pneumococcal Vaccination Status } \\
\hline Outcome & $\begin{array}{c}\text { Prior to admission } \\
(\mathbf{N}=\mathbf{8 5 7}) \\
\mathbf{n} / \mathbf{N}(\%)\end{array}$ & $\begin{array}{l}\text { During or after } \\
\text { admission } \\
(\mathbf{N}=\mathbf{8 0}) \\
\mathbf{n} / \mathbf{N}(\%)\end{array}$ & $\begin{array}{c}\text { Vaccination } \\
\text { not } \\
\text { documented } \\
(\mathbf{N}=499) \\
\mathbf{n} / \mathbf{N}(\%)\end{array}$ & $\begin{array}{c}\text { Overall } \\
(\mathrm{N}=1436) \\
\mathbf{n} / \mathbf{N}(\%)\end{array}$ & $\begin{array}{l}\text { p-value } \\
\text { p-value }^{\dagger} \\
\text { p-value }^{\ddagger}\end{array}$ \\
\hline
\end{tabular}

\section{Mortality}

Influenza vaccination status

$$
\text { Prior to admission }
$$

During or after admission

Vaccination not documented

Overall

$$
\begin{gathered}
52 / 350(14.9) \\
8 / 260(3.1) \\
11 / 247(4.5) \\
71 / 857(8.3)
\end{gathered}
$$

$1 / 15(6.7)$

$1 / 42(2.4)$

$1 / 23(4.3)$

$3 / 80(3.8)$

$52 / 138(37.7)$
$7 / 60(11.7)$
$75 / 301(24.9)$

134/499 (26.9)
105/503 (20.9)

$16 / 362(4.4)$

$87 / 571(15.2)$

208/1436 (14.5)

\section{Subsequent myocardial infarction}

Influenza vaccination status

Prior to admission

During or after admission

Vaccination not documented

Overall
10/350 (2.9)

10/260 (3.8)

10/247 (4.0)

$30 / 857(3.5)$
$1 / 15(6.7)$

$1 / 42(2.4)$

$1 / 23(4.3)$

$3 / 80(3.8)$
10/138 (7.2)

$4 / 60(6.7)$

23/301 (7.6)

$37 / 499(7.4)$
21/503 (4.2)

$0.01^{*}$

$15 / 362(4.1)$

$<0.01^{\dagger}$

$34 / 571(6.0)$

$0.32^{*}$

$\mathrm{n}$ denotes the number of patients with a given outcome; $\mathrm{N}$ denotes the number of patients cross-classified by pneumococcal and influenza vaccination status; "Based on 4 degree of freedom Fisher exact tests comparing the 9 time-dependent subgroups of patients by pneumococcal and influenza vaccination status; ${ }^{\dagger}$ Based on 2 degree of freedom Fisher exact tests comparing marginal distributions by pneumococcal vaccination status; ${ }^{\dagger}$ Based on 2 degree of freedom Fisher exact tests comparing marginal distributions by influenza vaccination status. 
Table 4. Unadjusted and adjusted associations between pneumococcal and influenza vaccination status, considered jointly, and outcomes at 6 months.

\begin{tabular}{|c|c|c|c|c|c|c|}
\hline \multirow[b]{2}{*}{ Model/method of adjustment } & \multicolumn{3}{|c|}{ Mortality } & \multicolumn{3}{|c|}{ Myocardial infarction } \\
\hline & HR & $(95 \%$ CI $)$ & p-value & HR & $(95 \%$ CI) & p-value \\
\hline \multicolumn{7}{|l|}{ Univariatemodels (Unadjusted) } \\
\hline Pneumococcal vaccination not documented & 1.0 & & & 1.0 & & \\
\hline Pneumococcal vaccination & 0.31 & $0.23-0.41$ & $<0.001$ & 0.50 & $0.31-0.80$ & $<0.01$ \\
\hline Influenza vaccination not documented & 1.0 & & & 1.0 & & \\
\hline Influenza vaccination & 1.90 & $1.44-2.51$ & $<0.001$ & 0.73 & $0.44-1.21$ & 0.22 \\
\hline \multicolumn{7}{|l|}{ Main effects model (Unadjusted) } \\
\hline Vaccination not documented & 1.0 & & & 1.0 & & \\
\hline Pneumococcal vaccination & 0.25 & $0.19-0.34$ & $<0.001$ & 0.52 & $0.32-0.84$ & $<0.01$ \\
\hline Influenza vaccination & 2.48 & $1.87-3.29$ & $<0.001$ & 0.83 & $0.50-1.38$ & 0.47 \\
\hline Interaction model (Unadjusted) & & & $<0.001^{*}$ & & & $0.03^{*}$ \\
\hline $\begin{array}{l}\text { Vaccination not documented } \\
\text { Pneumococcal vaccination only }\end{array}$ & $\begin{array}{l}1.0 \\
0.13\end{array}$ & $0.07-0.23$ & $<0.001$ & 0.58 & $0.32-1.03$ & 0.06 \\
\hline Influenza vaccination only & 1.88 & $1.33-2.64$ & $<0.001$ & 0.97 & $0.48-1.95$ & 0.93 \\
\hline Pneumococcal and influenza vaccinations & 0.66 & $0.47-0.92$ & 0.02 & 0.41 & $0.21-0.80$ & $<0.01$ \\
\hline Interaction model (Covariate adjusted) & & & $<0.001^{*}$ & & & $0.06^{*}$ \\
\hline $\begin{array}{l}\text { Vaccination not documented } \\
\text { Pneumococcal vaccination only }\end{array}$ & $\begin{array}{l}1.0 \\
0.11\end{array}$ & $0.06-0.20$ & $<0.001$ & 0.69 & $0.38-1.23$ & 0.21 \\
\hline Influenza vaccination only & 1.46 & $1.02-2.08$ & 0.04 & 0.91 & $0.45-1.83$ & 0.78 \\
\hline Pneumococcal and influenza vaccinations & 0.47 & $0.33-0.67$ & $<0.001$ & 0.40 & $0.20-0.79$ & $<0.01$ \\
\hline Age (per year) & 1.04 & $1.03-1.06$ & $<0.001$ & - & - & - \\
\hline Systolic blood pressure $<90 \mathrm{~mm} \mathrm{Hg}$ & 1.63 & $1.19-2.24$ & $<0.01$ & - & - & - \\
\hline Pulmonary edema on admission & 1.77 & $1.25-2.49$ & 0.001 & 1.80 & $0.99-3.26$ & 0.05 \\
\hline Hemoglobin $<11.5 \mathrm{gm} / \mathrm{dl}$ & 2.20 & $1.65-2.93$ & $<0.001$ & - & - & - \\
\hline Left ventricular ejection fraction $<35 \%$ & - & - & - & 2.16 & $1.21-3.84$ & 0.02 \\
\hline Missing & - & - & - & 1.08 & $0.62-1.91$ & \\
\hline Current or past smoker & 0.76 & $0.57-1.02$ & 0.07 & - & - & - \\
\hline Troponin elevated & 1.48 & $1.10-1.99$ & 0.01 & 3.66 & $2.26-5.91$ & $<0.001$ \\
\hline Diabetes mellitus & 1.33 & $1.00-1.77$ & 0.05 & - & - & - \\
\hline Statin use & 0.45 & $0.32-0.62$ & $<0.001$ & & & \\
\hline Missing & 0.83 & $0.56-1.22$ & 0.35 & - & - & - \\
\hline $\begin{array}{l}\text { Interaction model (Propensity score adju } \\
\text { forpneumococcal vaccination) }\end{array}$ & & & $<0.001^{*}$ & & & $0.07^{*}$ \\
\hline $\begin{array}{l}\text { Vaccination not documented } \\
\text { Pneumococcal vaccination only }\end{array}$ & $\begin{array}{l}1.0 \\
0.12\end{array}$ & $0.06-0.21$ & $<0.001$ & 0.60 & $0.32-1.10$ & 0.10 \\
\hline Influenza vaccination only & 1.82 & $1.29-2.58$ & $<0.01$ & 1.02 & $0.51-2.08$ & 0.95 \\
\hline Pneumococcal and influenza vaccinations & 0.60 & $0.42-0.85$ & $<0.01$ & 0.44 & $0.22-0.89$ & 0.02 \\
\hline
\end{tabular}




\section{Continued}

\begin{tabular}{ccccccc}
\hline $\begin{array}{c}\text { Interaction model (Propensity score adjustment for } \\
\text { influenza vaccination) } \\
\text { Vaccination not documented }\end{array}$ & 1.0 & & $<0.001^{*}$ & & $<0.01^{*}$ \\
Pneumococcal vaccination only & 0.11 & $0.06-0.20$ & $<0.001$ & 0.46 & $0.25-0.85$ & 0.01 \\
$\quad$ Influenza vaccination only & 1.71 & $1.20-2.41$ & $<0.01$ & 0.84 & $0.41-1.71$ & 0.63 \\
Pneumococcal and influenza vaccinations & 0.53 & $0.37-0.75$ & $<0.001$ & 0.33 & $0.16-0.66$ & $<0.01$ \\
\hline
\end{tabular}

HR denotes hazard ratio; CI denotes confidence interval; ${ }^{*}$ These p-values assess differences in hazard ratios across the 4 time-dependent subgroups of patients defined by pneumococcal and influenza; vaccination status (considered jointly), based on 3 degree of freedom tests of heterogeneity.

pneumococcal vaccination only and HR of 0.66 (95\% CI 0.47 - 0.92) for both vaccinations, while patients with influenza vaccination only had a significantly higher risk of mortality (HR $=1.88 ; 95 \%$ CI $1.33-2.64)$.

As shown in Figure 1(b) and summarized in Table 4, patients with both vaccinations had a significantly lower risk of subsequent $\mathrm{MI}(\mathrm{HR}=0.41 ; 95 \%$ CI $0.21-0.80)$ and those with pneumococcal vaccination only had a borderline significantly lower risk of subsequent MI (HR $=0.58 ; 95 \%$ CI $0.32-1.03)$ compared to patients with neither vaccination. Risk of subsequent MI was similar for patients with influenza vaccination only and patients with neither vaccination $(\mathrm{HR}=0.97 ; 95 \% \mathrm{CI} 0.48-1.95)$.

\subsection{Adjusted Associations between Vaccination and Patient Outcomes}

After covariate adjustment for the variables shown in Table 4, the adjusted HRs were similar to the corresponding unadjusted HRs for both mortality and subsequent MI. The overall test for heterogeneity of mortality by vaccination status was statistically significant for both the unadjusted and adjusted models ( $p<0.001$ for each), and the corresponding tests for subsequent MI were significant in the unadjusted analysis $(\mathrm{p}=0.03)$ and of borderline statistical significance $(\mathrm{p}=0.06)$ in the adjusted analysis.

Similar associations between pneumococcal and influenza vaccination status and mortality were obtained when the analyses were adjusted for the propensity to receive pneumococcal vaccination or the propensity to receive influenza vaccination (Table 4). Consistently across quintiles of propensity scores for pneumococcal vaccination (Figure 2(a)) and influenza vaccination (Figure 2(b)), mortality was lowest for patients who received pneumococcal vaccination only and highest for patients who received influenza vaccination only.

Similar associations between pneumococcal and influenza vaccination and subsequent MI were obtained after adjustment for the propensity to receive pneumococcal or the propensity to receive influenza vaccination (Table 4). However, for patients with pneumococcal vaccination only, the risk of subsequent MI was signifi- cantly lower only after adjustment for the propensity to receive influenza vaccination $(\mathrm{HR}=0.46 ; 95 \%$ CI 0.25 $0.85)$. For all three methods of adjustment, patients who received influenza vaccination only had a risk of subsequent MI similar to that of unvaccinated patients, and patients who received both vaccines had significantly reduced risk of subsequent $\mathrm{MI}$.

\section{Discussion}

In our prospective cohort of 1436 patients hospitalized with suspected ACS/NSTEMI, receipt of pneumococcal vaccine alone or combined with influenza vaccination were associated with statistically significant lower mortality at 6 months. Receipt of both pneumococcal and influenza vaccination was associated with a significantly decreased risk of subsequent MI at 6 months, and pneumococcal vaccination alone was associated with a borderline significant lower risk of this outcome. The mortality benefits were observed among patients presenting with as well as without a NSTEMI, confirmed using troponin-I levels, and persisted after adjusting for potential confounders using both multivariable adjustment and two types of propensity score adjustment. The observed risk reduction for mortality among recipients of pneumococcal vaccination is striking given that patients who received pneumococcal vaccination were significantly older and had a greater burden of co morbid conditions, such as diabetes and hypertension.

Although the role of pneumococcal vaccination in preventing the most commonly caused bacterial form of pneumonia is well-documented, its role in preventing in-hospital mortality and respiratory failure as well as decreasing length of stay in patients with community acquired pneumonia shows mixed results [14-16]. Its role in patients presenting with ACS has remained largely unexplored. In a recent case-control study [17], patients who had received prior pneumococcal vaccine were at a $50 \%$ lower risk of presenting with an MI. This study, unlike ours, was not designed to assess mortality. Although our study does show a lower risk of subsequent MI, the effect size was not as substantial as that reported 


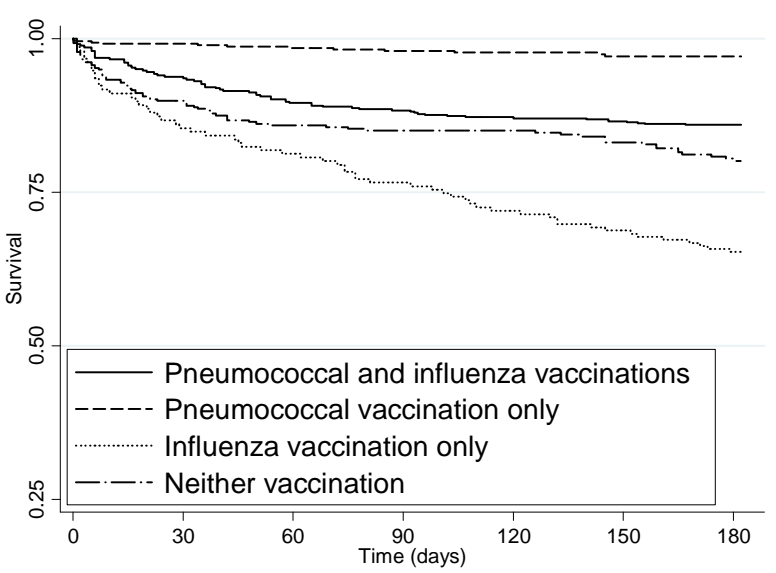

(a)

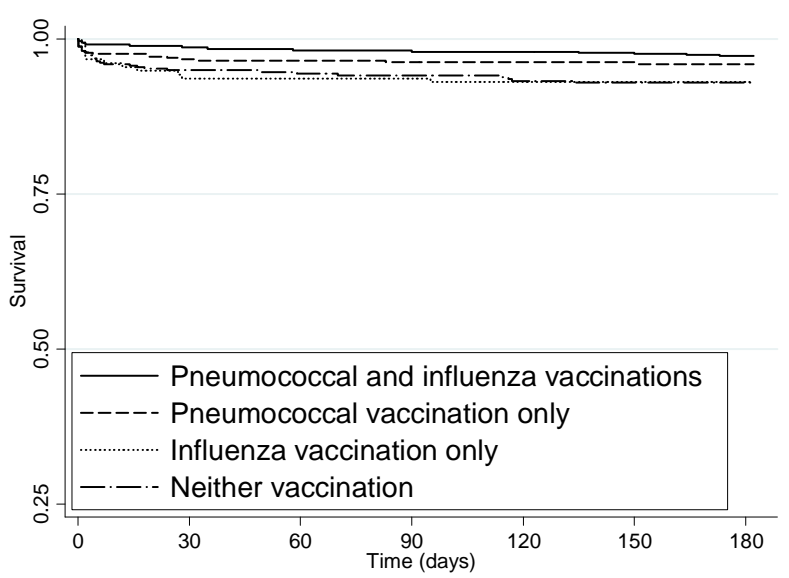

(b)

Figure 1. Kaplan-meier estimated survival for time to (a) death and (b) myocardial infarction within 6 months of admission by time-dependent pneumococcal and influenza vaccination status. Solid lines denote both pneumococcal and influenza vaccinations, dashed line denote pneumococcal vaccination only; dotted lines denote influenza vaccination only; and long dashed and dotted lines denote neither vaccination. (a) Time to death within 6 months; (b) Time to subsequent myocardial infarction within 6 months.

by Lamontagne et al. [17]. In contrast, Tseng et al. [18] failed to find an association between receipt of pneumococcal vaccination and reduced risk of MI or stroke in men. One possible explanation is that the Tseng et al. study was based on a relatively younger population with no prior documented cardiovascular disease and a much lower risk profile than patients in the present study. Our study population was a high-risk, older population with high prevalence of co-morbidities who presented with suspected ACS and had a relatively high rate of adverse outcomes within 6 months. Our finding of an apparent beneficial effect of pneumococcal vaccination was unexpected. We also did not expect a significant interaction between pneumococcal and influenza vaccination status in terms of all-cause mortality within 6 months. Patients who had received only influenza vaccination fared worse than non-vaccinated patients in terms of mortality (unadjusted HR $=1.88$ ), while patients with pneumococcal vaccination only fared the best (unadjusted $H R=0.13$ ). Receipt of both vaccinations appears to be associated with an intermediate benefit (HR of 0.66). This interaction was unexpected and contrary to prior studies [12,23], reporting a beneficial effect in patients presenting with an MI or an ACS. However neither of these studies addressed potential confounding by history of pneumococcal vaccination. In our cohort of patients with suspected ACS, many who received pneumococcal vaccination also received influenza vaccination (71.2\%), making it difficult to isolate the effect of each type of vaccination. More complete data on timing and indications for both types of vaccination are needed to investigate this further.

A few recent studies of the combined effect of influenza and pneumococcal vaccination on preventing ad- verse cardiovascular outcomes have reported conflicting results $[24,25]$. In the Siriwardena et al. study of 16,012 post-MI patients matched with 62,694 controls, receipt of influenza vaccination was associated with a $19 \%$ reduction in the risk of subsequent MI while no beneficial effect was noted with pneumococcal vaccination [25]. Hung et al. reported that receipt of both vaccines, but neither one alone, was associated with significant reductions in all-cause mortality and cardiovascular outcomes in a cohort of elderly persons [24]. Both of these studies were population based, with populations at lower risk than our fairly high-risk group of Veterans who presented with a suspected ACS and had a 6month combined outcome rate of nearly $20 \%$.

Our study has several limitations. First, conducting our study at a single VA medical center with a predominantly Caucasian, male patient population limits its generalizibility. Second, we focused on a subset of patients with cardiovascular disease, specifically those admitted with suspected ACS/NSTEMI. Although ACS/NSTEMI currently comprises a majority of patients presenting with chest pain, the results may not be applicable to patients presenting with ST-segment elevation MI or other cardiovascular disorders such as stable angina, peripheral vascular disease, or congestive heart failure. Third, any observational study is vulnerable to confounding by unmeasured factors. Although our results were virtually unchanged by propensity score adjustment for receipt of either pneumococcal vaccination or influenza vaccination, propensity scores cannot adjust for unmeasured confounders that are uncorrelated with measured confounders. Finally, we did not have data on the incidence 


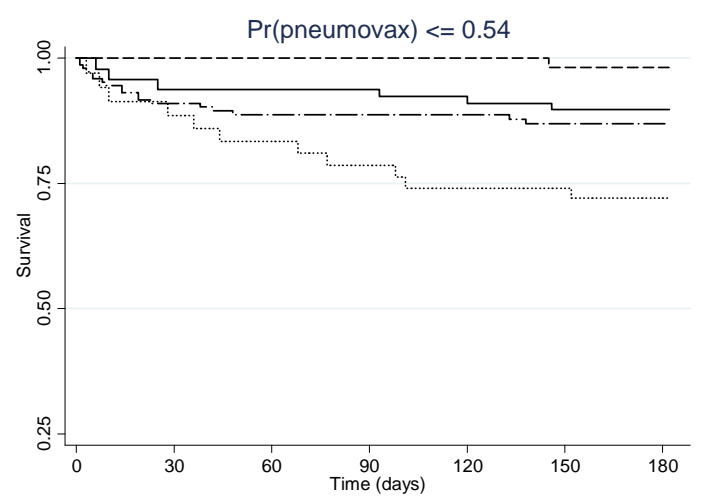

(a)

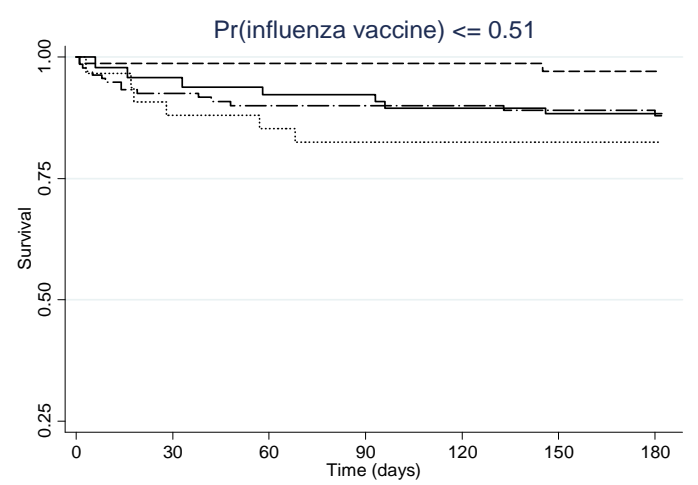

(b)

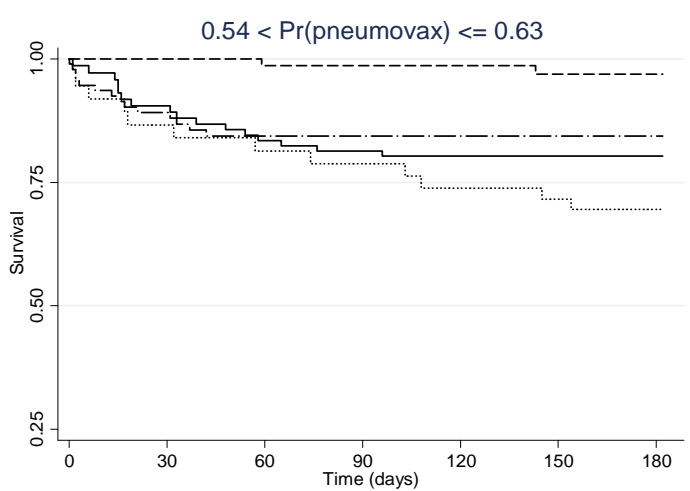

(c)

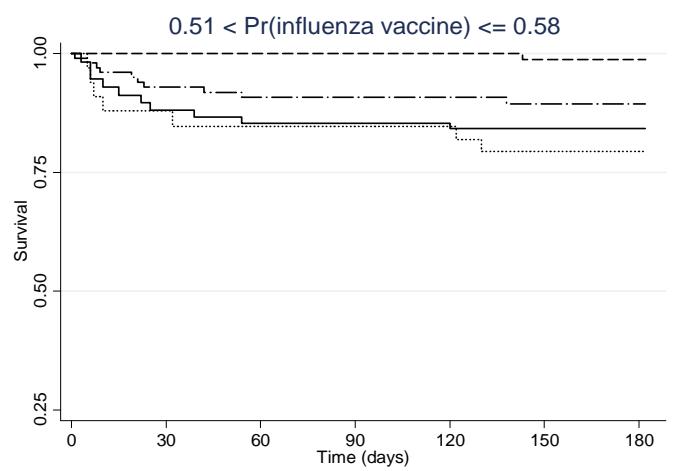

(d)

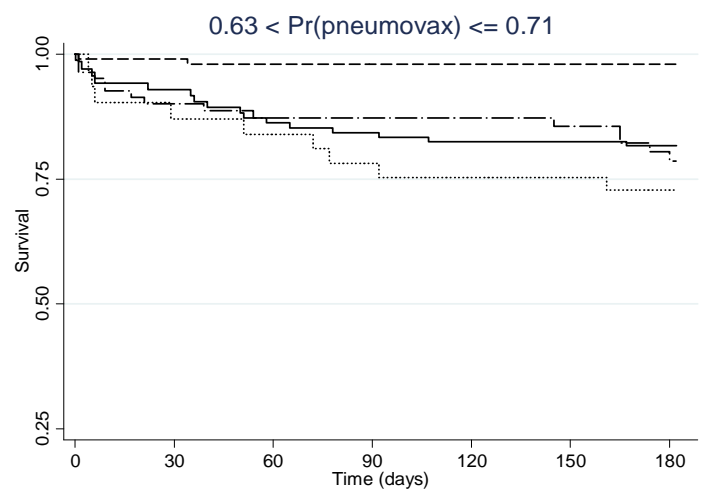

(e)

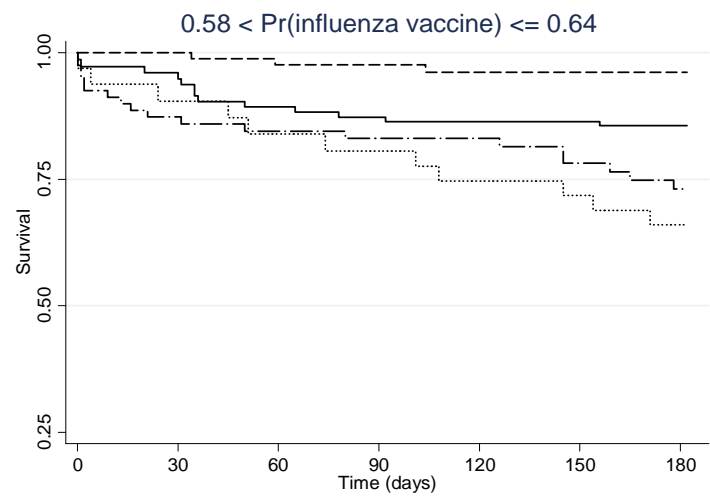

(f)

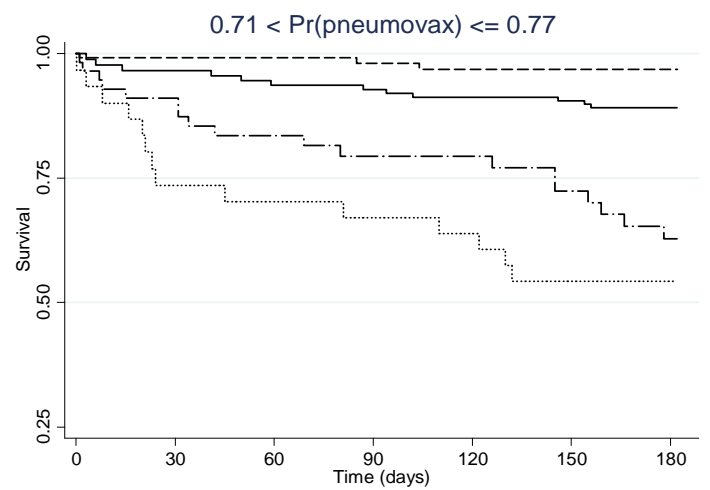

(g)

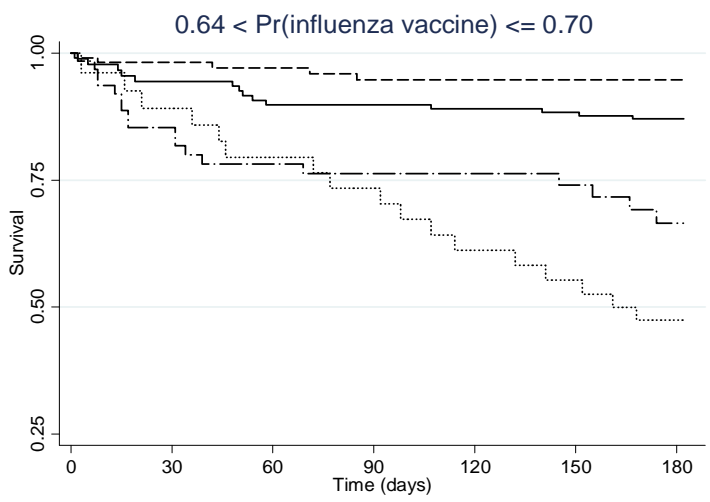

(h) 


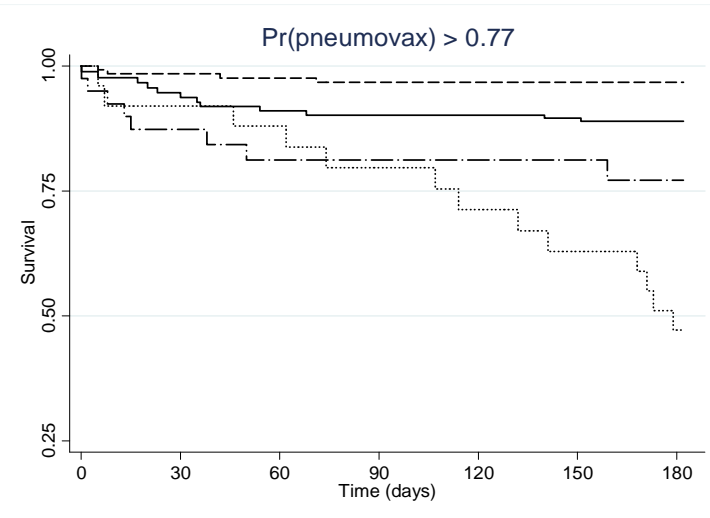

(i)

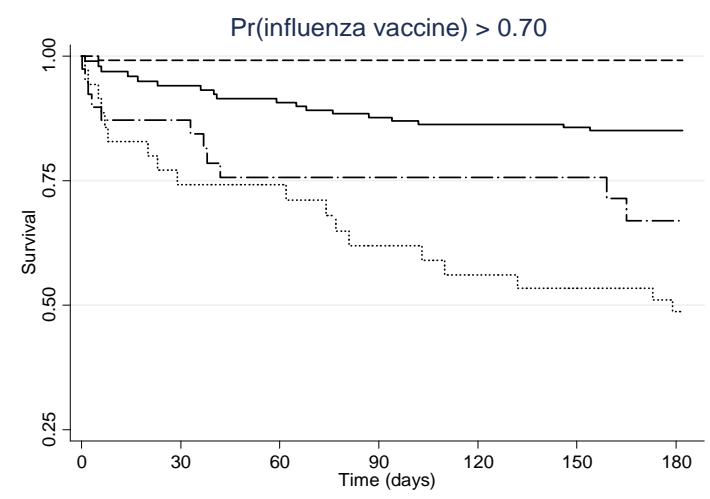

(j)

Figure 2. Kaplan-meier estimated survival for time to death within 6 months of admission by time-dependent pneumococcal and influenza vaccination status. Analyses are stratified by quintile of the propensity scores for (a) pneumococcal vaccination and (b) influenza vaccination. Solid lines denote both pneumococcal and influenza vaccinations, dashed lines denote pneumococcal vaccination only, dotted lines denote influenza vaccination only, and long dashed and dotted lines denote neither vaccination; pneumococcal propensity score quintiles Influenza propensity score quintiles.

of acute respiratory illnesses, influenza or pneumococcal infections prior to the index admission or during the follow up period, or systematic ascertainment of MIs that occurred outside the VA hospital system. We could not estimate cause-specific mortality rates, as some deaths were identified by reviewing VA national databases that include applications for Veteran death benefits, which do not include cause of death. A relatively large number of patients had missing data on left ventricular ejection fraction or statin use and it was not possible to distinguish between in-hospital and discharge medications.

In summary, our data suggest that pneumococcal vaccination is associated with reduced risk of death among patients admitted with suspected ACS/NSTEMI. Our findings should be interpreted as provocative but pre- liminary, and require confirmation in other databases and large randomized clinical trials before any statement can be made about the clinical utility of pneumococcal vaccination in preventing adverse cardiovascular events in patients presenting with a suspected ACS.

\section{Conclusion}

Among patients hospitalized with suspected ACS, pneumococcal vaccination, with or without influenza vaccination, was associated with significantly lower risk of mortality within 6 months. A similar trend in the risk of subsequent MI did not reach statistical significance. Our results remained unchanged after adjustment for receipt of influenza vaccination as well as after propensity score adjustments. Our findings are provocative but should be considered preliminary. Future studies, including those using a randomized controlled design, would be important to confirm our provocative findings.

\section{REFERENCES}

[1] F. A. Spencer, R. J. Goldberg, R. C. Becker and J. M. Gore, "Seasonal Distribution of Acute Myocardial Infarction in the Second National Registry of Myocardial Infarction," Journal of the American College of Cardiology, Vol. 31, No. 6, 1998, pp. 1226-1233. doi:10.1016/S0735-1097(98)00098-9

[2] N. Rumana, Y. Kita, T. C. Turin, Y. Murakami, H. Sugihara, et al., "Seasonal Pattern of Incidence and Case Fatality of Acute Myocardial Infarction in a Japanese Population (from the Takashima AMI Registry, 1988 to 2003)," American Journal of Cardiology, Vol. 102, No. 10, 2008, pp. 1307-1311. doi:10.1016/j.amjcard.2008.07.005

[3] L. Smeeth, S. L. Thomas, A. J. Hall, R. Hubbard, P. Farrington, et al., "Risk of Myocardial Infarction and Stroke after Acute Infection or Vaccination," The New England Journal of Medicine, Vol. 351, No. 25, 2004, pp. 26112618. doi:10.1056/NEJMoa041747

[4] G. N. Fredrikson, B. Hedblad, G. Berglund, R. Alm, M. Ares, et al., "Identification of Immune Responses against Aldehyde-Modified Peptide Sequences in Apob Associated with Cardiovascular Disease," Arteriosclerosis, Thrombosis, and Vascular Biology, Vol. 23, No. 5, 2003, pp. 872-878. doi:10.1161/01.ATV.0000067935.02679.B0

[5] K. J. Mattila, V. V. Valtonen, M. S. Nieminen and S. Asikainen, "Role of Infection as a Risk Factor for Atherosclerosis, Myocardial Infarction, and Stroke," Clinical Infectious Diseases, Vol. 26, No. 3 1998, pp. 719-734. doi: $10.1086 / 514570$

[6] H. Uzui, A. Harpf, M. Liu, T. M. Doherty, A. Shukla, et al., "Increased Expression of Membrane Type 3-Matrix Metalloproteinase in Human Atherosclerotic Plaque: Role of Activated Macrophages and Inflammatory Cytokines," Circulation, Vol. 106, No. 24, 2002, pp. 3024-3030. doi:10.1161/01.CIR.0000041433.94868.12

[7] C. Buono, C. J. Binder, G. Stavrakis, J. L. Witztum, L. H. 
Glimcher, et al., "T-Bet Deficiency Reduces Atherosclerosis and Alters Plaque Antigen-Specific Immune Responses," Proceedings of the National Academy of Sciences of the United States of America, Vol. 102, No. 5, 2005, pp. 1596-1601. doi:10.1073/pnas.0409015102

[8] P. K. Shah, K. Y. Chyu, G. N. Fredrikson and J. Nilsson, "Immunomodulation of Atherosclerosis with a Vaccine," Nature Clinical Practice Cardiovascular Medicine, Vol. 2, No. 12 2005, pp. 639-646. doi:10.1038/ncpcardio0372

[9] C. P. Cannon, E. Braunwald, C. H. McCabe, J. T. Grayston, B. Muhlestein, et al., "Antibiotic Treatment of Chlamydia Pneumoniae after Acute Coronary Syndrome," The New England Journal of Medicine, Vol. 352, No. 16, 2005 , pp. 1646-1654. doi:10.1056/NEJMoa043528

[10] J. B. Muhlestein, J. L. Anderson, J. F. Carlquist, K. Salunkhe, B. D. Horne, et al., "Randomized Secondary Prevention Trial of Azithromycin in Patients with Coronary Artery Disease: Primary Clinical Results of the Academic Study," Circulation, Vol. 102, No. 15, 2000, pp. 1755-1760. doi:10.1161/01.CIR.102.15.1755

[11] M. Naghavi, Z. Barlas, S. Siadaty, S. Naguib, M. Madjid, et al., "Association of Influenza Vaccination and Reduced Risk of Recurrent Myocardial Infarction," Circulation, Vol. 102, No. 25, 2000, pp. 3039-3045. doi:10.1161/01.CIR.102.25.3039

[12] E. P. Gurfinkel, R. L. de la Fuente, O. Mendiz and B. Mautner, "Influenza Vaccine Pilot Study in Acute Coronary Syndromes and Planned Percutaneous Coronary Interventions: The FLU Vaccination Acute Coronary Syndromes (FLUVACS) Study," Circulation, Vol. 105, No. 18, 2002, pp. 2143-2147. doi:10.1161/01.CIR.0000016182.85461.F4

[13] C. J. Binder, S. Horkko, A. Dewan, M. K. Chang, E. P. Kieu, et al., "Pneumococcal Vaccination Decreases Atherosclerotic Lesion Formation: Molecular Mimicry between Streptococcus Pneumoniae and Oxidized LDL," Nature Medicine, Vol. 9, No. 6, 2003, pp. 736-743. doi: $10.1038 / \mathrm{nm} 876$

[14] S. A. Moberley, J. Holden, D. P. Tatham and R. M. Andrews, "Vaccines for Preventing Pneumococcal Infection in Adults," Cochrane Database of Systematic Reviews, 2008.

[15] A. Huss, P. Scott, A. E. Stuck, C. Trotter and M. Egger, "Efficacy of Pneumococcal Vaccination in Adults: A MetaAnalysis," Canadian Medical Association Journal, Vol. 180, No. 1, 2009, pp. 48-58. doi:10.1503/cmaj.080734

[16] M. J. Fine, M. A. Smith, C. A. Carson, F. Meffe, S. S. Sankey, et al., "Efficacy of Pneumococcal Vaccination in Adults. A Meta-Analysis of Randomized Controlled Trials," Archives of Internal Medicine, Vol. 154, No. 23, 1994, pp. 2666-2677.
[17] D. M. Musher, A. M. Rueda, A. S. Kaka and S. M. Mapara, "The Association between Pneumococcal Pneumonia and Acute Cardiac Events," Clinical Infectious Diseases, Vol. 45, No. 2, 2007, pp. 158-165. doi:10.1086/518849

[18] V. F. Corrales-Medina, K. N. Suh, G. Rose, J. A. Chirinos, S. Doucette, et al., "Cardiac Complications in Patients with Community-Acquired Pneumonia: A Systematic Review and Meta-Analysis of Observational Studies," PLoS Medicine, Vol. 8, 2011, p. e1001048.

[19] F. Lamontagne, M. P. Garant, J. C. Carvalho, L. Lanthier, M. Smieja, et al., "Pneumococcal Vaccination and Risk of Myocardial Infarction," Canadian Medical Association Journal, Vol. 179, No. 8, 2008, pp. 773-777. doi:10.1503/cmaj.070221

[20] H. F. Tseng, J. M. Slezak, V. P. Quinn, L. S. Sy, S. K. Van den Eeden, et al., "Pneumococcal Vaccination and Risk of Acute Myocardial Infarction and Stroke in Men," The Journal of the American Medical Association, Vol. 303, No. 17, 2010, pp. 1699-1706. doi:10.1001/jama.2010.529

[21] J. P. Nuorti and C. G. Whitney, "Updated Recommendations for Prevention of Invasive Pneumococcal Disease among Adults Using the 23-Valent Pneumococcal Polysaccharide Vaccine (PPS V23)," Morbidity and Mortality Weekly Report, Vol. 59, No. 34, 2010, pp. 1102-1106.

[22] C. P. Cannon, A. Battler, R. G. Brindis, J. L. Cox, S. G. Ellis, et al., "American College of Cardiology Key Data Elements and Definitions for Measuring the Clinical Management and Outcomes of Patients with Acute Coronary Syndromes. A Report of the American College of Cardiology Task Force on Clinical Data Standards (Acute Coronary Syndromes Writing Committee)," Journal of the American College of Cardiology, Vol. 38, No. 7, 2001, pp. 2114-2130.

[23] A. Phrommintikul, S. Kuanprasert, W. Wongcharoen, R. Kanjanavanit, R. Chaiwarith, et al., "Influenza Vaccination Reduces Cardiovascular Events in Patients with Acute Coronary Syndrome," European Heart Journal, Vol. 32, No. 14, 2011, pp. 1730-1735. doi:10.1093/eurheartj/ehr004

[24] I. F. Hung, A. Y. Leung, D. W. Chu, D. Leung, T. Cheung, et al., "Prevention of Acute Myocardial Infarction and Stroke among Elderly Persons by Dual Pneumococcal and Influenza Vaccination: A Prospective Cohort Study," Clinical Infectious Diseases, Vol. 51, No. 9, 2010, pp. 10071016. doi:10.1086/656587

[25] A. N. Siriwardena, S. M. Gwini and C. A. Coupland, "Influenza Vaccination, Pneumococcal Vaccination and Risk of Acute Myocardial Infarction: Matched Case-Control Study," Canadian Medical Association Journal, Vol. 182, No. 15, 2010, pp. 1617-1623. doi:10.1503/cmaj.091891 